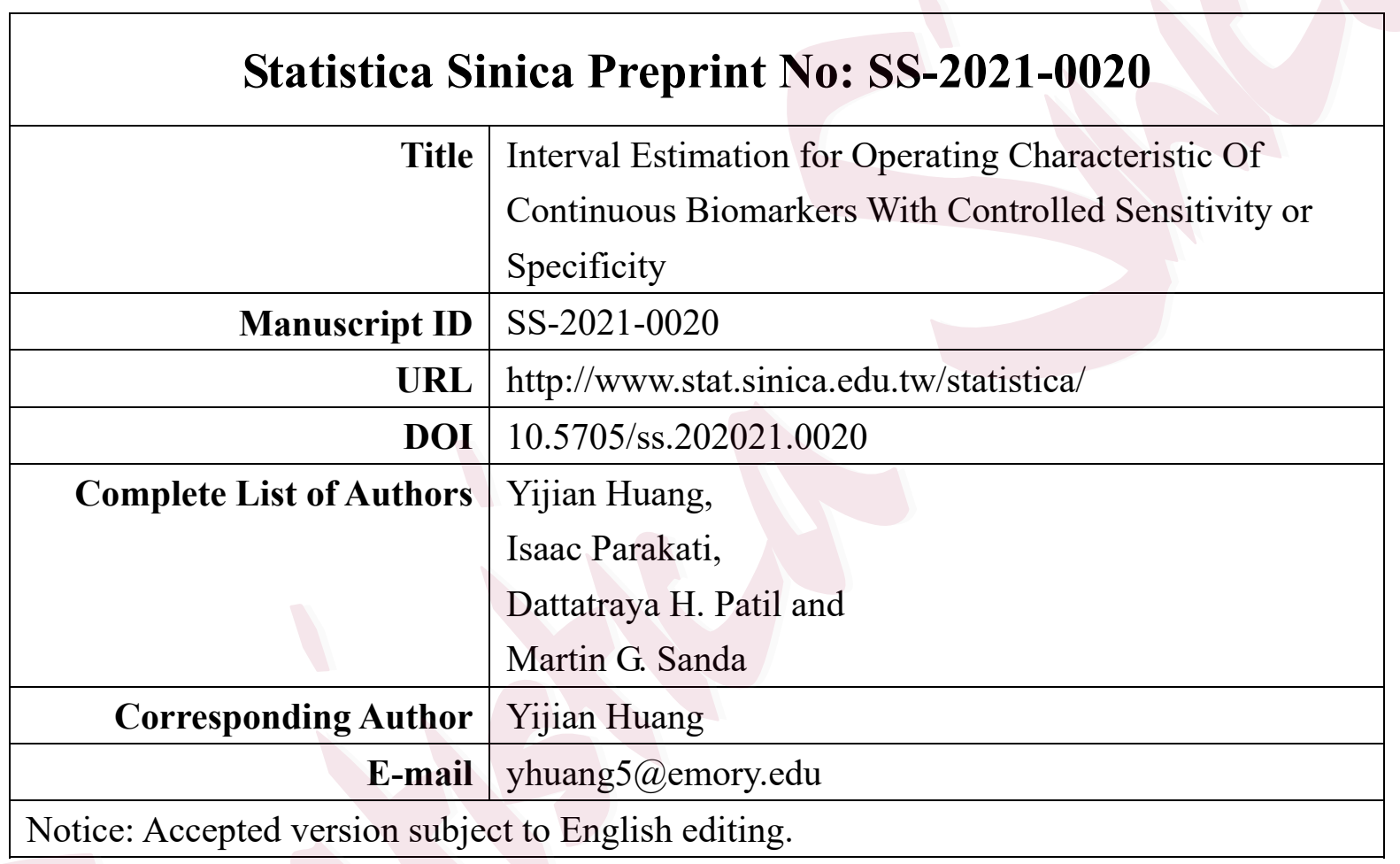


Statistica Sinica

\title{
Interval estimation for operating characteristic of continuous biomarkers with controlled sensitivity or specificity
}

\author{
Yijian Huang ${ }^{1}$, Isaac Parakati², \\ Dattatraya H. Patil ${ }^{1}$, and Martin G. Sanda ${ }^{1}$ \\ ${ }^{1}$ Emory University \\ and ${ }^{2}$ Ann $\&$ Robert H. Lurie Children's Hospital of Chicago
}

Abstract: The receiver operating characteristic (ROC) curve provides a comprehensive performance assessment of a continuous biomarker over the full threshold spectrum. Nevertheless, a medical test often dictates to operate at a certain high level of sensitivity or specificity. A diagnostic accuracy metric directly targeting the clinical utility is specificity at the controlled sensitivity level, or vice versa. While the empirical point estimation is readily adopted in practice, the nonparametric interval estimation is challenged by the fact that the variance involves density functions due to estimated threshold. In addition, even with a fixed threshold, many standard confidence intervals including the Wald interval for binomial proportion could have erratic behaviors. In this article, we are motivated by the superior performance of the score interval for binomial proportion and propose a novel extension for the biomarker problem. Meanwhile, 
we develop exact bootstrap and establish consistency of the bootstrap variance estimator. Both single-biomarker evaluation and two-biomarker comparison are investigated. Extensive simulation studies were conducted, demonstrating competitive performance of our proposals. An illustration with aggressive prostate cancer diagnosis is provided.

Key words and phrases: Diagnostic test, exact bootstrap, score confidence interval, sensitivity at controlled specificity, specificity at controlled sensitivity.

\section{Introduction}

Fueled by the rapid recent advances in the scientific knowledge of molecular biology and high-throughput omics technologies, a large number of candidate biomarkers for disease diagnosis, prognosis, and prediction of response to specific therapeutic interventions have been or are being identified in this era of precision medicine. Biomarker evaluation and comparison has become ever more important for their validation and further clinical translation that would ultimately lead to improvement and advance of clinical practice (e.g., Tzoulaki et al., 2011; Ioannidis and Panagiotou, 2011). Many biomarkers are continuous, and their dichotomization at a threshold is necessary for clinical testing as being binary. Sensitivity and specificity vary with the threshold, giving rise to the receiver operating characteristic (ROC) curve. While the ROC curve fully characterizes the performance of 
a biomarker over the complete threshold spectrum, only the point where the test is intended to operate is clinically relevant. For example, with aggressive prostate cancer diagnosis, a positive non-invasive test would be confirmed by biopsy. As a result, the cost of a false negative greatly outweighs that of a false positive. In this circumstance, the non-invasive test needs to attain a high sensitivity, say, 95\% (e.g., Catalona et al., 1998; Sanda et al., 2017) to be clinically useful. Therefore, specificity at the controlled sensitivity level would be a more sensible accuracy metric than, say, area under the ROC curve which is popular in practice. Of course, sensitivity at a controlled specificity level could be more relevant in a different clinical context. Nevertheless, the two correspond to the very same statistical problem, upon transposing the roles of cases and controls. We shall focus on the former throughout this article.

While the empirical estimator of specificity at a controlled sensitivity level is straightforward to obtain, the nonparametric interval estimation is complicated by the fact that the variance involves density functions of the biomarker for case and control populations (cf. Linnett, 1987; Pepe, 2003). Pepe (2003) suggested to use kernel smoothing for the density estimation. However, this approach can be sensitive to the bandwidth choice, and choosing an appropriate bandwidth is often challenging with practi- 
cal sample sizes. Furthermore, the approach is not invariant to monotone transformation of the biomarker. As an alternative, Platt et al. (2000) and Zhou and Qin (2005) proposed to adopt resampling bootstrap. Nevertheless, the uncertainty from resampling affects reproducibility, despite that the error can be made small with increasing resampling size.

If the threshold is fixed, the problem of concern reduces to the interval estimation for binomial proportion. Nevertheless, even with this basic problem, many standard confidence intervals have erratic behaviors (Agresti and Coull, 1998; Brown et al., 2001, 2002). In particular, the simple and widelyused Wald interval tends to have considerable under-coverage. Meanwhile, the score interval (Wilson, 1927) is recognized for its superior performance. Agresti and Coull (1998) suggested an adjusted Wald interval mimicking the score interval to achieve better coverage performance. These results have influenced the interval estimation for our problem of concern, i.e., with estimated threshold. Specifically, Zhou and Qin (2005) incorporated the Agresti-Coull adjustment in their proposals, although the justification does not seem completely clear in this new context.

In this article, we propose a novel extension of the score interval for binomial proportion to specificity at a controlled sensitivity level. As another contribution, we develop exact bootstrap and establish consistency 
of the bootstrap variance estimator. In Section 2, we focus on the evaluation of a single biomarker. Section 3 is devoted to the comparison of two biomarkers, under both unpaired and paired designs (cf. Pepe, 2003). A bias analysis of the empirical specificity at controlled sensitivity is provided in Section 4, leading to an alternative point estimate and subsequently the associated confidence intervals. Simulations are reported in Section 5, and an illustration given in Section 6 with prostate cancer detection. Final remarks are provided in Section 7. Technical details including proofs are relegated to the Appendix. An $\mathrm{R}$ package that implements the proposed methods is publicly available at the first author's website http: //web1.sph. emory.edu/users/yhuang5.

\section{Proposed method for single-biomarker evaluation}

Consider a biomarker of interest $M$. Denote the case and control variables by $M_{\bullet}$ and $M_{\circ}$, respectively. Write their distribution functions as $F_{\bullet}(t) \equiv \operatorname{Pr}\left(M_{\bullet} \leq t\right)$ and $F_{\circ}(t) \equiv \operatorname{Pr}\left(M_{\circ} \leq t\right)$, and the quantile function of the former as $F_{\bullet}^{-1}(p) \equiv \inf \left\{t: F_{\bullet}(t) \geq p\right\}$. The case sample consists of $n_{\bullet}$ independent replicates of $M_{\bullet}: M_{\bullet}, i=1, \cdots, n_{\bullet}$, whereas the control sample comprises $n_{\circ}$ independent replicates of $M_{\circ}: M_{\circ i}, i=1, \cdots, n_{\circ}$. Adopt the convention that reaching or exceeding a given threshold results in a 
positive diagnosis. With $\rho_{0} \in(0,1)$ as the controlled level of sensitivity, the largest threshold is $\tau_{0}=F_{\bullet}^{-1}\left\{\left(1-\rho_{0}\right)+\right\}$ such that the sensitivity defined as $\operatorname{Pr}\left(M_{\bullet} \geq \tau_{0}\right)$ is at least $\rho_{0}$. Accordingly, the specificity is $\phi_{0} \equiv F_{\circ}\left(\tau_{0}-\right)$. Their natural plug-in estimators are given by

$$
\widehat{\tau}=\widehat{F}_{\bullet}^{-1}\left\{\left(1-\rho_{0}\right)+\right\}, \quad \widehat{\phi}=\widehat{F}_{\circ}(\widehat{\tau}-),
$$

where $\widehat{F}_{\bullet}$ and $\widehat{F}_{\circ}$ are the empirical versions of $F_{\bullet}$ and $F_{\circ}$, respectively. Under regularity conditions, $\widehat{\phi}$ is asymptotically normal with mean $\phi_{0}$ and variance

$$
\sigma^{2}=\sigma_{1}^{2}+\sigma_{2}^{2} \equiv\left\{\frac{F_{\circ}^{\prime}\left(\tau_{0}\right)}{F_{\bullet}^{\prime}\left(\tau_{0}\right)}\right\}^{2} \frac{\rho_{0}\left(1-\rho_{0}\right)}{n_{\bullet}}+\frac{\phi_{0}\left(1-\phi_{0}\right)}{n_{\circ}}
$$

where $F_{\bullet}^{\prime}$ and $F_{\circ}^{\prime}$ are the derivatives of $F_{\bullet}$ and $F_{\circ}$, respectively; see Greenhouse and Mantel (1950), Hsieh and Turnbull (1996), and Pepe (2003), among others, and also Theorem 1 as presented later. The variance has two components, $\sigma_{1}^{2}$ resulting from the threshold estimation and $\sigma_{2}^{2}$ from the empirical specificity with given threshold $\tau_{0}$. As discussed in Section 1 , the involvement of density functions complicates the variance estimation.

\subsection{Exact bootstrap}

Bootstrap is an effective approach to the variance estimation. Platt et al. (2000) and Zhou and Qin (2005) suggested the routine resampling implementation. To improve reproducibility, we develop exact bootstrap 
and show its feasibility for this problem.

Focus first on the threshold estimation with the cases. Maritz and Jarrett (1978) and Efron (1979) derived the exact bootstrap distribution for a sample order statistic. From their result, we obtain a resampling scheme that is equivalent to the bootstrap resampling with respect to a sample quantile. Denote the ceiling function by $\lceil\cdot\rceil$.

Lemma 1. Consider an iid sample of a random variable with size $n$. Denote the empirical cumulative distribution function by $\widehat{F}$ and its bootstrap counterpart by $F^{*}$. For any $p \in(0,1)$ and conditional on the observed data, $F^{*-1}(p)$ has the same distribution as $\widehat{F}^{-1}(B)$ where independent random variable $B$ follows $\operatorname{Beta}(\lceil n p\rceil, n-\lceil n p\rceil+1)$.

Remark 1. This lemma was deduced from Maritz and Jarrett (1978) and Efron (1979). However, it might become more intuitive in light of a representation of the distribution of $\widehat{F}^{-1}(p)$. That is, $\widehat{F}^{-1}(p)$ as an order statistic can be shown to have the same distribution as $F^{-1}(B)$, where $F$ is the underlying cumulative distribution function under consideration.

This result does not impose any restriction on the underlying distribution, which can be continuous, discrete, or a mixture of the two. Write $\tau^{*}$ as the bootstrap counterpart of $\widehat{\tau}$. Given that $\widehat{\tau}$ is the $\left\{\left(1-\rho_{0}\right)+\right\}$ quantile, the bootstrap distribution of $\tau^{*}$ then is the same as $\widehat{F}_{\bullet}^{-1}\left(1-B_{\bullet}\right)$, 
where $B_{\bullet} \sim \operatorname{Beta}\left(n_{\bullet}-r+1, r\right)$ and $r \equiv\left\lceil n_{\bullet}\left(1-\rho_{0}\right)+\right\rceil$. That is, the bootstrap distribution assigns to order statistics $M_{\bullet}[i], i=1, \cdots, n_{\bullet}$, with the same probabilities as $1-B$ • to the $n_{\bullet}$ intervals evenly split between 0 and 1 . This resampling equivalence facilitates efficient computation with exact bootstrap. Moreover, this novel perspective of $\tau^{*}$ can be exploited in large-sample study as shown later.

Now, turn to the specificity estimation with the controls, at given threshold $\tau^{*}$. Let $\phi^{*}$ be the bootstrap counterpart of $\widehat{\phi}$. Write $\operatorname{Pr}^{*}$ as the bootstrap probability, i.e., conditional on the observed data. The conditional bootstrap probability mass function is

$$
\operatorname{Pr}^{*}\left(\phi^{*}=\phi \mid \tau^{*}\right)=\left(\begin{array}{c}
n_{\circ} \\
n_{\circ} \phi
\end{array}\right) \widehat{F}_{\circ}\left(\tau^{*}-\right)^{n_{\circ} \phi}\left\{1-\widehat{F}_{\circ}\left(\tau^{*}-\right)\right\}^{n_{\circ}(1-\phi)},
$$

for $\phi \in\left\{0,1 / n_{\circ}, \cdots,\left(n_{\circ}-1\right) / n_{\circ}, 1\right\}$. Upon rescaling by $n_{\circ}$, this is a binomial distribution with size $n_{\circ}$ and success probability $\widehat{F}_{\circ}\left(\tau^{*}-\right)$.

As the case and control samples are independent of each other, combining the preceding results accordingly gives the bootstrap distribution of the specificity at controlled sensitivity $\rho_{0}$ :

$$
\operatorname{Pr}^{*}\left(\phi^{*}=\phi\right)=\sum_{i=1}^{n \bullet} \operatorname{Pr}^{*}\left(\tau^{*}=M_{\bullet[i]}\right) \operatorname{Pr}^{*}\left(\phi^{*}=\phi \mid \tau^{*}=M_{\bullet[i]}\right),
$$

for $\phi \in\left\{0,1 / n_{\circ}, \cdots,\left(n_{\circ}-1\right) / n_{\circ}, 1\right\}$. This exact bootstrap distribution is feasible to compute, although care is needed to avoid numerical underflow 
and overflow.

Write $E_{*}$ and $\operatorname{Var}_{*}$ as conditional expectation and variance given the observed data, respectively. The bootstrap variance estimator of $\widehat{\phi}$ is $\operatorname{Var}_{*}\left(\phi^{*}\right)$ :

$$
\begin{aligned}
\widehat{\sigma}^{2} & =\operatorname{Var}_{*}\left\{\widehat{F}_{\circ}\left(\tau^{*}-\right)\right\}+E_{*}\left[n_{\circ}^{-1} \widehat{F}_{\circ}\left(\tau^{*}-\right)\left\{1-\widehat{F}_{\circ}\left(\tau^{*}-\right)\right\}\right] \\
& \equiv \widehat{\sigma}_{1}^{2}+\widehat{\sigma}_{2}^{2}
\end{aligned}
$$

which are estimators of $\sigma_{1}^{2}$ and $\sigma_{2}^{2}$, respectively, as components of $\sigma^{2}$ given in $(2.1)$.

The consistency and asymptotic normality of $\widehat{\phi}$ have long been known; see, for example, Greenhouse and Mantel (1950). However, theoretical justification may not have been provided even for consistency of the bootstrap distribution, and much less for that of the bootstrap variance estimator; in general, the former does not necessarily imply the latter (e.g., Ghosh et al., 1984; Shao, 1990). The following result focuses on the bootstrap distribution and variance. Nevertheless, the asymptotic properties of $\widehat{\phi}$ are also stated mostly for completeness, with weaker assumptions imposed and a proof from which the consistency of the bootstrap distribution can immediately follow.

Theorem 1. Suppose that the following conditions hold: (i) size ratio of cases and controls $n_{\bullet} / n_{\circ}$ converges to a non-zero finite constant as $n_{\bullet}+n_{\circ}$ 
2.2 Confidence intervals

approaches $\infty$, (ii) $\rho_{0} \in(0,1)$, and (iii) $F_{\bullet}$ and $F_{\circ}$ are differentiable at the threshold $\tau_{0}$ with $F_{\bullet}^{\prime}\left(\tau_{0}\right)>0$. Then, $\widehat{\phi}$ is strongly consistent for $\phi_{0}$, and asymptotically normal with mean $\phi_{0}$ and variance $\sigma^{2}$ as given in (2.1).

Meanwhile, $n_{\circ}^{1 / 2}\left(\phi^{*}-\widehat{\phi}\right)$ conditional on the data converges in distribution to the same limit as $n_{\circ}^{1 / 2}\left(\widehat{\phi}-\phi_{0}\right)$. Furthermore, under the additional condition (iv) $F_{\bullet}$ and $F_{\circ}$ are continuously differentiable in a neighborhood around $\tau_{0}$, $n_{\bullet} \widehat{\sigma}_{1}^{2}, n_{\circ} \widehat{\sigma}_{2}^{2}$, and subsequently $n_{\circ} \widehat{\sigma}^{2}$ converge in probability to $n_{\bullet} \sigma_{1}^{2}, n_{\circ} \sigma_{2}^{2}$, and $n_{\circ} \sigma^{2}$, respectively.

\subsection{Confidence intervals}

With the bootstrap variance estimator $\widehat{\sigma}^{2}$, a Wald $100(1-\alpha) \%$ confidence interval is given by $\widehat{\phi} \pm z_{\alpha / 2} \widehat{\sigma}$, where $z_{\alpha / 2}$ is the $(\alpha / 2)$-quantile of the standard normal distribution. The interval is truncated with $[0,1]$ to respect the parameter range. Another common and simple interval is the percentile $100(1-\alpha) \%$ confidence interval, as the interval between the $\alpha / 2$ and $(1-\alpha / 2)$-quantiles of the bootstrap distribution.

We further propose a novel confidence interval. For binomial proportion, Agresti and Coull (1998) and Brown et al. (2001, 2002) among others showed that the score interval (Wilson, 1927) has good coverage accuracy even for very small sample size. It outperforms many other competitors 
including the Wald interval and the "exact" interval of Clopper and Pearson (1934). Like the Wald interval, the score interval is inverted from a hypothesis test. But the difference is that the score interval adopts the null variance. Unfortunately, the estimated specificity at controlled sensitivity is rather the proportion of an overdispersed binomial due to the estimated threshold. Therefore, its variance is not fully determined by the null specificity. We overcome this issue by estimating the overdispersion factor with $\widehat{\sigma}^{2} \widehat{\sigma}_{2}^{-2}$. The resulting score interval is given by

$$
\left\{\phi: \frac{(\widehat{\phi}-\phi)^{2}}{n_{\circ}^{-1} \phi(1-\phi) \widehat{\sigma}^{2} \widehat{\sigma}_{2}^{-2}}<z_{\alpha / 2}^{2}\right\},
$$

which has an explicit expression with the two bounds as solutions to a quadratic equation. Since the denominator approaches 0 as $\phi$ goes to 0 or 1 , this interval is guaranteed to be contained in the parameter range $[0,1]$.

All these three confidence intervals are invariant to monotone transformation of the biomarker. Thanks to the exact bootstrap, they are also perfectly reproducible.

\section{Two-biomarker comparison}

Comparison of two biomarkers, say $X$ and $Y$, is also of common interest in practice. Denote the quantities in Section 2 associated with each biomarker by adding subscript "X" or "Y". At the common controlled sensi- 


\subsection{Unpaired comparison}

tivity $\rho_{0}$, the specificity difference $\delta_{0} \equiv \phi_{0 X}-\phi_{0 Y}$ between the two provides a meaningful measure for their clinical utility difference. With estimated thresholds $\widehat{\tau}_{X}$ and $\widehat{\tau}_{Y}$, the corresponding estimated specificities $\widehat{\phi}_{X}$ and $\widehat{\phi}_{Y}$ and subsequently their estimated difference $\widehat{\delta}=\widehat{\phi}_{X}-\widehat{\phi}_{Y}$ are obtained. This point estimation procedure remains the same whether the two biomarkers are measured in two independent studies or the same one, i.e., under unpaired or paired design, respectively (cf. Pepe, 2003). But the inference would be different, and also more complicated.

\subsection{Unpaired comparison}

With the two biomarkers measured in independent studies, the bootstrap distributions of $\widehat{\phi}_{X}$ and $\widehat{\phi}_{Y}$ are independent of each other. Then, the bootstrap variance estimator and also distribution of the difference in specificity $\widehat{\delta}$ can be easily obtained. Subsequently, the Wald and percentile confidence intervals for $\delta_{0}$ are constructed in the same fashion as for the single-biomarker evaluation in Section 2.2.

Nevertheless, it is unclear how to construct a score interval. To follow the approach for the single-biomarker evaluation, the two null specificities at the controlled sensitivity would be needed. However, they are not determined except for their difference $\delta$. We suggest instead combining $\delta$ and the 
other biomarker's estimated specificity, i.e., $\delta+\widehat{\phi}_{Y}$ and $\widehat{\phi}_{X}-\delta$ as estimated specificities under the null for biomarkers $X$ and $Y$, respectively. To this end, we propose the following score confidence interval for $\delta_{0}$ :

$$
\begin{array}{r}
{\left[\delta:(\widehat{\delta}-\delta)^{2}<z_{\alpha / 2}^{2}\left\{\frac{\widehat{\sigma}_{X}^{2}}{n_{\circ X} \widehat{\sigma}_{2 X}^{2}}\left(\delta+\widehat{\phi}_{Y}\right)\left(1-\delta-\widehat{\phi}_{Y}\right)\right.\right.} \\
\left.\left.+\frac{\widehat{\sigma}_{Y}^{2}}{n_{\circ Y} \widehat{\sigma}_{2 Y}^{2}}\left(\widehat{\phi}_{X}-\delta\right)\left(1-\widehat{\phi}_{X}+\delta\right)\right\}\right] .
\end{array}
$$

Different from the more standard form as in (2.5), the variance component in the above display is moved to the other side of the inequality. Since $\delta+\widehat{\phi}_{Y}$ and $\widehat{\phi}_{X}-\delta$ are not guaranteed to be bounded between 0 and 1 , the variance component may not necessarily be positive. The current form is more sensible since a negative variance component is against, rather than for, the null. Just like (2.5) for single biomarker evaluation, the confidence interval (3.6) has an explicit expression with the two bounds being the solutions to a quadratic equation; existence of the solutions can be easily shown. The interval is truncated by $[-1,1]$ to respect the parameter range.

\subsection{Paired comparison}

Denote the pair of biomarkers by $\left(X_{\bullet}, Y_{\bullet}\right)^{\top}$ for a case and $\left(X_{\circ}, Y_{\circ}\right)^{\top}$ for a control. The case sample consists of $n_{\bullet}$ independent replicates, $\left(X_{\bullet i}, Y_{\bullet}\right)^{\top}$, $i=1, \cdots, n_{\bullet}$, and the control sample of $n_{\circ}$ independent replicates, $\left(X_{\circ i}, Y_{\circ i}\right)^{\top}$, $i=1, \cdots, n_{\circ}$. 
To derive the exact bootstrap distribution, we start with the cases. For $s, t=0,1$, introduce

$$
\widehat{m}_{\bullet s t}(x, y)=\#\left\{I\left(X_{\bullet i} \leq x\right)=s, I\left(Y_{\bullet i} \leq y\right)=t: i=1, \cdots, n_{\bullet}\right\}
$$

Write $\widehat{\mathbf{m}}_{\bullet}(x, y)=\left\{\widehat{m}_{\bullet 11}(x, y), \widehat{m}_{\bullet 10}(x, y), \widehat{m}_{\bullet 01}(x, y), \widehat{m}_{\bullet 00}(x, y)\right\}^{\top}$ and $\mathbf{m}_{\bullet}^{*}(x, y)=$ $\left\{m_{\bullet 11}^{*}(x, y), m_{\bullet 10}^{*}(x, y), m_{\bullet 01}^{*}(x, y), m_{\bullet 00}^{*}(x, y)\right\}^{\top}$ as its bootstrap counterpart. As $\left(\tau_{X}^{*}, \tau_{Y}^{*}\right)^{\top}$ may take a value only in $\Omega=\left\{X_{\bullet i}: i=1, \cdots, n_{\bullet}\right\} \times\left\{Y_{\bullet} ;:\right.$ $\left.j=1, \cdots, n_{\bullet}\right\}$, we have $\operatorname{Pr}^{*}\left(\tau_{X}^{*} \leq X_{\bullet i}, \tau_{Y}^{*} \leq Y_{\bullet j}\right)=\operatorname{Pr}^{*}\left\{m_{\bullet 11}^{*}\left(X_{\bullet i}, Y_{\bullet j}\right)+m_{\bullet 10}^{*}\left(X_{\bullet i}, Y_{\bullet j}\right) \geq r\right.$,

$$
\left.m_{\bullet 11}^{*}\left(X_{\bullet i}, Y_{\bullet j}\right)+m_{\bullet 01}^{*}\left(X_{\bullet i}, Y_{\bullet j}\right) \geq r\right\}
$$

recall $r \equiv\left\lceil n_{\bullet}\left(1-\rho_{0}\right)+\right\rceil$. The right-hand side above can be calculated from the fact that

$$
\mathbf{m}_{\bullet}^{*}\left(X_{\bullet}, Y_{\bullet j}\right) \mid \text { observed data } \sim \operatorname{Multinomial}\left\{n_{\bullet}, \widehat{\mathbf{m}}_{\bullet}\left(X_{\bullet}, Y_{\bullet}\right) / n_{\bullet}\right\}
$$

Now with the controls, we derive the bootstrap distribution of $\delta^{*}$, the bootstrap counterparts of $\widehat{\delta}$, conditional on the thresholds $\tau_{X}^{*}$ and $\tau_{Y}^{*}$. In parallel to their case counterparts, introduce

$$
\widehat{m}_{\circ s t}(x, y)=\#\left\{I\left(X_{\circ i} \leq x\right)=s, I\left(Y_{\circ i} \leq y\right)=t: i=1, \cdots, n_{\circ}\right\}
$$

for $s, t=0,1$, and subsequently $\widehat{\mathbf{m}}_{\circ}(x, y)$ and $\mathbf{m}_{\circ}^{*}(x, y)$. It is clear that

$$
\mathbf{m}_{\circ}^{*}\left(\tau_{X}^{*}, \tau_{Y}^{*}\right) \mid \text { observed data, } \tau_{X}^{*}, \tau_{Y}^{*} \sim \operatorname{Multinomial}\left\{n_{\circ}, \widehat{\mathbf{m}}_{\circ}\left(\tau_{X}^{*}, \tau_{Y}^{*}\right) / n_{\circ}\right\}
$$


from which the bootstrap distribution of $\delta^{*}$ given $\tau_{X}^{*}$ and $\tau_{Y}^{*}$ can be obtained. For that purpose, $\delta^{*}=\left\{m_{\circ 10}^{*}\left(\tau_{X}^{*}, \tau_{Y}^{*}\right)-m_{\circ 01}^{*}\left(\tau_{X}^{*}, \tau_{Y}^{*}\right)\right\} / n_{\circ}$ as $\widehat{\delta}=$ $\left\{\widehat{m}_{\circ 10}\left(\widehat{\tau}_{X}, \widehat{\tau}_{Y}\right)-\widehat{m}_{\circ 01}\left(\widehat{\tau}_{X}, \widehat{\tau}_{Y}\right)\right\} / n_{\circ}$

Combining the preceding results gives the bootstrap distribution of $\delta^{*}$. The bootstrap variance estimator of $\widehat{\delta}$ is given by

$$
\begin{aligned}
\widehat{\sigma}_{\delta}^{2}=\operatorname{Var}_{*}\left\{E_{*}\left(\delta^{*} \mid \tau_{X}^{*}, \tau_{Y}^{*}\right)\right\}+E_{*}\left\{\operatorname{Var}_{*}\left(\delta^{*} \mid \tau_{X}^{*}, \tau_{Y}^{*}\right)\right\} \\
=\sum_{(x, y)^{\top} \in \Omega}\left[\left\{n_{\circ}^{-1} \widehat{m}_{\circ 10}(x, y)-n_{\circ}^{-1} \widehat{m}_{\circ 01}(x, y)-E_{*} \delta^{*}\right\}^{2}\right. \\
+n_{\circ}^{-2} \widehat{m}_{\circ 10}(x, y)\left\{1-n_{\circ}^{-1} \widehat{m}_{\circ 10}(x, y)\right\} \\
+n_{\circ}^{-2} \widehat{m}_{\circ 01}(x, y)\left\{1-n_{\circ}^{-1} \widehat{m}_{\circ 01}(x, y)\right\} \\
\left.+2 n_{\circ}^{-3} \widehat{m}_{\circ 10}(x, y) \widehat{m}_{\circ 01}(x, y)\right] \operatorname{Pr}^{*}\left(\tau_{X}^{*}=x, \tau_{Y}^{*}=y\right),
\end{aligned}
$$

where $E_{*} \delta^{*}=\sum_{(x, y)^{\top} \in \Omega} n_{\circ}^{-1}\left\{\widehat{m}_{\circ 10}(x, y)-\widehat{m}_{\circ 01}(x, y)\right\} \operatorname{Pr}^{*}\left(\tau_{X}^{*}=x, \tau_{Y}^{*}=y\right)$. This computation is obviously more intensive than that for the singlebiomarker evaluation, but still feasible.

Theorem 2. Suppose that at least one of the two correlations, between $I\left(X_{\bullet} \leq \tau_{0 X}\right)$ and $I\left(Y_{\bullet} \leq \tau_{0 Y}\right)$ and between $I\left(X_{\circ} \leq \tau_{0 X}\right)$ and $I\left(Y_{\circ} \leq \tau_{0 Y}\right)$, is less than 1. Under conditions (i), (ii), and (iii) as for each biomarker in Theorem 1, conditional on the data, $n_{\circ}^{1 / 2}\left(\delta^{*}-\widehat{\delta}\right)$ converges in distribution to the same limit as $n_{\circ}^{1 / 2}\left(\widehat{\delta}-\delta_{0}\right)$, which is normal with mean zero. 
If condition (iv) in Theorem 1 holds additionally for each biomarker, $n_{\circ} \widehat{\sigma}_{\delta}^{2}$ converges in probability to the asymptotic variance of $n_{\circ}^{1 / 2}\left(\widehat{\delta}-\delta_{0}\right)$.

Asymptotic degeneracy of the joint distribution of $\widehat{\phi}_{X}$ and $\widehat{\phi}_{Y}$ is avoided with the above correlation condition.

With the bootstrap variance estimator and bootstrap distribution of $\widehat{\delta}$, the Wald and percentile confidence intervals can be constructed for $\delta_{0}$. For the score confidence interval, we build upon that for unpaired comparison as given in (3.6) by further accounting for the difference between $\widehat{\sigma}_{\delta}^{2}$ and $\widehat{\sigma}_{X}^{2}+\widehat{\sigma}_{Y}^{2}:$

$$
\begin{aligned}
& {\left[\delta:(\widehat{\delta}-\delta)^{2}<\frac{z_{\alpha / 2}^{2} \widehat{\sigma}_{\delta}^{2}}{n_{\circ}\left(\widehat{\sigma}_{X}^{2}+\widehat{\sigma}_{Y}^{2}\right)}\left\{\frac{\widehat{\sigma}_{X}^{2}}{\widehat{\sigma}_{2 X}^{2}}\left(\delta+\widehat{\phi}_{Y}\right)\left(1-\delta-\widehat{\phi}_{Y}\right)\right.\right.} \\
& \left.\left.+\frac{\widehat{\sigma}_{Y}^{2}}{\widehat{\sigma}_{2 Y}^{2}}\left(\widehat{\phi}_{X}-\delta\right)\left(1-\widehat{\phi}_{X}+\delta\right)\right\}\right] .
\end{aligned}
$$

Like the previous ones, this score confidence interval also has an explicit expression with the two bounds as solutions to a quadratic equation. Also, the interval is truncated by $[-1,1]$ to respect the parameter range.

\section{Alternative point estimator for improved performance}

Focus on the single-biomarker evaluation as in Section 2. The estimated threshold is the $\left\lceil n \cdot\left(1-\rho_{0}\right)+\right\rceil$-th order statistic of the cases. Due to the discreteness nature, intuitively the bias of $\widehat{\phi}$ would have an oscillating com- 
ponent with variable $n_{\bullet}$. A more formal analysis given in the Appendix shows that

$$
\begin{aligned}
E(\widehat{\phi})-\phi_{0}= & \frac{F_{\circ}^{\prime}\left(\tau_{0}\right)}{F_{\bullet}^{\prime}\left(\tau_{0}\right)}\left(\frac{\left\lceil n_{\bullet}\left(1-\rho_{0}\right)+\right\rceil}{n_{\bullet}+1}-1+\rho_{0}\right) \\
& +\left\{\frac{F_{\circ}^{\prime \prime}\left(\tau_{0}\right)}{F_{\bullet}^{\prime}\left(\tau_{0}\right)^{2}}-\frac{F_{\circ}^{\prime}\left(\tau_{0}\right) F_{\bullet}^{\prime \prime}\left(\tau_{0}\right)}{F_{\bullet}^{\prime}\left(\tau_{0}\right)^{3}}\right\} \frac{\rho_{0}\left(1-\rho_{0}\right)}{2 n_{\bullet}}+o\left(n_{\bullet}^{-1}\right),
\end{aligned}
$$

provided $F_{\bullet}$ being continuous and strictly increasing and the existence and continuity of the second derivatives $F_{\bullet}^{\prime \prime}$ and $F_{\circ}^{\prime \prime}$, in a neighborhood of $\tau_{0}$. This bias result is sharper than that of Lloyd and Yong (1999, theorem 2). While both bias terms are of order $n_{\bullet}^{-1}$, the first one is the oscillating component. For example, with fixed $\rho_{0}=0.95$, the first term vanishes whenever $0.05\left(n_{\bullet}+1\right)$ is an integer, which occurs at increments of 20 .

This bias analysis suggests an alternative point estimator free of this oscillating bias component. Write $\lfloor\cdot\rfloor$ as the floor function. Consider two threshold estimates as the $\left\lfloor\left(n_{\bullet}+1\right)\left(1-\rho_{0}\right)\right\rfloor$ - and $\left\lceil\left(n_{\bullet}+1\right)\left(1-\rho_{0}\right)\right\rceil$-th order statistics of the case biomarkers. If the two order statistics are the same, they lead to the estimated specificity $\widehat{\phi}$, since $\left\lfloor\left(n_{\bullet}+1\right)\left(1-\rho_{0}\right)\right\rfloor \leq$ $\left\lceil n_{\bullet}\left(1-\rho_{0}\right)+\right\rceil \leq\left\lceil\left(n_{\bullet}+1\right)\left(1-\rho_{0}\right)\right\rceil$. Otherwise, obtain the weighted average of the empirical specificities at these two thresholds as $\widetilde{\phi}$, with weights $\left\lceil\left(n_{\bullet}+1\right)\left(1-\rho_{0}\right)\right\rceil-\left(n_{\bullet}+1\right)\left(1-\rho_{0}\right)$ and $\left(n_{\bullet}+1\right)\left(1-\rho_{0}\right)-\left\lfloor\left(n_{\bullet}+1\right)\left(1-\rho_{0}\right)\right\rfloor$, 
respectively. It is straightforward to show

$$
E(\widetilde{\phi})-\phi_{0}=\left\{\frac{F_{\circ}^{\prime \prime}\left(\tau_{0}\right)}{F_{\bullet}^{\prime}\left(\tau_{0}\right)^{2}}-\frac{F_{\circ}^{\prime}\left(\tau_{0}\right) F_{\bullet}^{\prime \prime}\left(\tau_{0}\right)}{F_{\bullet}^{\prime}\left(\tau_{0}\right)^{3}}\right\} \frac{\rho_{0}\left(1-\rho_{0}\right)}{2 n_{\bullet}}+o\left(n_{\bullet}^{-1}\right) .
$$

The two estimators, $\widehat{\phi}$ and $\widetilde{\phi}$, are asymptotically equivalent to each other to the first order and they coincide when $\left(n_{\bullet}+1\right)\left(1-\rho_{0}\right)$ is an integer. This construction is reminiscent of the usual definition of sample median, which is the average of the two middle order statistics in the case of even sample size. Indeed, it can be applied to the threshold estimation instead. But we would not do so since the resulting specificity estimator would no longer be invariant to monotone transformation of the biomarker.

By replacing $\widehat{\phi}$ with $\widetilde{\phi}$, alternatives to the Wald and score confidence intervals in Section 2.2 are obtained. The variance components are kept the same, although the exact bootstrap for $\widetilde{\phi}$ may be developed. This very same approach also leads to new Wald and score intervals for two-biomarker comparison as discussed in Section 3.

Note that $\widetilde{\phi}$ still shares the same non-oscillating bias component of order $n_{\bullet}^{-1}$ with $\widehat{\phi}$. It is possible to further develop an estimator that is unbiased to order $n_{\bullet}^{-1}$ via, for example, delete- $d$ jackknife. However, this bias reduction may not result in a reduction in mean squared error. In fact, preliminary numerical studies did not show performance improvement in the resulting confidence intervals. Therefore, we did not pursue further. 


\section{Simulations}

Extensive simulations have been conducted to evaluate the proposed methods under practical sample sizes. Throughout, the controlled sensitivity level was set to $95 \%$ and the nominal level of confidence intervals to 95\%. Under each set-up, 1000 replications were simulated. Set-ups with equal case and control sizes are reported herewith, and others are included in the supplementary material; results were largely similar.

\subsection{Single-biomarker evaluation}

For comparison, we included several existing confidence intervals. One of them employed kernel smoothing for density estimation to construct a Wald interval. To preserve the parameter range, this interval for logittransformed specificity was first formulated and then back-transformed, as described in Pepe (2003). The univariate adaptive kernel density estimation of Silverman (1986), as implemented in function ajk() of R package Quantreg with default tuning parameters, was adopted. Unlike other confidence intervals as being studied, this kernel-smoothing approach is not invariant to monotone transformation of the biomarker. For that reason, we also applied the method to the same data but after exponential transformation of the biomarker, since biomarkers are often non-negative and 
5.1 Single-biomarker evaluation

have skewed distributions in practice. Among confidence intervals based on resampling bootstrap, Zhou and Qin (2005) reported that their "BTII" interval performed better. This Zhou-Qin interval, as referred to herein, was implemented with two resampling sizes, 200 and 500. Size 200 is usually considered sufficient for Wald confidence intervals in general (cf. Efron and Tibshirani, 1994, section 6.4), whereas size 500 was adopted in the simulations of Zhou and Qin (2005). Additionally, we also constructed this interval based on exact bootstrap, equivalent to the case of infinite resampling size.

Both the case and control biomarkers followed the normal distributions of unity variance, but with different means to achieve a specified specificity at the controlled 95\% sensitivity. In the simulations, the original Zhou-Qin interval showed considerable variability from the resampling. Nevertheless, this variability was not reflected in coverage probability and averaged length. For this reason, only the exact bootstrap version is included in the reporting; the resampling variability can be seen later in Section 6. Figure 1 shows the coverage probabilities and averaged lengths of these confidence intervals over a grid of $\phi_{0}$ between 0.1 and 0.9 with mesh size 0.005 , in the setting of the case and control sample sizes $\left(n_{\bullet}, n_{\circ}\right)$ being $(50,50)$. The kernel smoothing method could be sensitive to the scale on which a biomarker is measured. With both of the scales considered, these intervals tended to be 
5.1 Single-biomarker evaluation

much wider and more conservative than the others. The exact bootstrapbased Wald and percentile intervals had similar averaged length, shorter than the kernel smoothing ones but longer than the Zhou-Qin and score intervals. However, the Wald intervals had considerable under coverage except at small $\phi_{0}$, whereas the percentile interval had coverage always above the nominal level. Overall, the Zhou-Qin and score intervals performed the best, reaching nominal coverage level with the shortest averaged length over most part of $\phi_{0}$. Between them, the score intervals tended to have shorter length in the middle value range of $\phi_{0}$, whereas the Zhou-Qin interval was tighter at the extremes.

These confidence intervals were also evaluated with fixed $\phi_{0}=0.2$, $0.4,0.6$, and 0.8 , and variable sample size $n_{\bullet}=n_{\circ}$ from 20 to 200 . The three best performers, the two score intervals using $\widehat{\phi}$ and $\widetilde{\phi}$ and ZhouQin interval, are reported in Figure 2. Except for the case of $\phi_{0}=0.8$, the Zhou-Qin interval had an oscillating pattern in coverage probability apparently due to the bias of the adopted point estimate $\widehat{\phi}$ as discussed in Section 4, and considerable under coverage could arise at certain sample sizes. Not surprisingly, this occurred to the score interval using $\widehat{\phi}$ as well. In contrast, the coverage probability of the score interval using $\widetilde{\phi}$ was much more stable with little oscillation. 
5.2 Two-biomarker comparison

Many of these confidence intervals are closely related to those for binomial proportion (Agresti and Coull, 1998; Brown et al., 2001). Nevertheless, the behavior patterns appeared to be different, at least when the case and control sizes were comparable. Oscillation in coverage could arise, but mainly due to the way that the threshold is estimated.

\subsection{Two-biomarker comparison}

We report unpaired comparison studies with $n_{\bullet X}=n_{\circ X}=n_{\bullet Y}=n_{\circ Y}$ and paired comparison ones with $n_{\bullet}=n_{\circ}$; results with other sample size set-ups were similar. Case and control sizes of 50 and 200 were considered. With unpaired comparison, each biomarker was simulated in the same fashion as in Section 5.1. Under paired comparison, $\left(X_{\circ}, Y_{\circ}\right)^{\top}$ followed the standard bivariate normal distribution with 0.5 correlation coefficient, and $\left(X_{\bullet}, Y_{\bullet}\right)^{\top}$ had a location shift from that distribution to attain specified specificity at controlled $95 \%$ sensitivity for each biomarker.

Table 1 shows the coverage probabilities and averaged lengths of the exact bootstrap-based Wald, percentile, and score confidence intervals for difference in specificity at controlled 95\% sensitivity. All the confidence intervals were reasonably close to the nominal level, but they all tended to be conservative when the sample size was smaller. The two score in- 
tervals, without and with oscillating bias-correction for the point estimate, were similar, both being considerably shorter than the other three, for both unpaired and paired comparisons alike.

The kernel smoothing-based Wald interval can be extended for the twobiomarker comparison. However, it was not included in our study in light of its less competitive performance in the single-biomarker evaluation. On the other hand, the Zhou-Qin interval may be extended as well. We studied its exact bootstrap version although the results are not included in the table. The Zhou-Qin interval also tended to be conservative. It was slightly shorter than the Wald and percentile intervals, but much wider than the score ones.

\section{Illustration with aggressive prostate cancer detection}

This development was motivated by prostate cancer research, to evaluate biomarkers for the detection of aggressive prostate cancer, i.e., Gleason score $\geq 7$, among men undergoing their first-time biopsy. Two biomarkers of interest are serum prostate health index (phi) and urine PCA3. A total of 512 participants enrolled from four urology groups affiliated with three academic medical centers consisted of 155 cases and 357 controls, per pathology testing on prostate biopsies (Sanda et al., 2017). They provided 
post-urinary specimens after digital rectal examination and serum specimens both before biopsy, and had their phi and PCA3 assayed. The metric of specificity at $95 \%$ sensitivity was adopted for the evaluation of biomarker performance.

Figure 3 shows the analysis results. To reach $95 \%$ sensitivity, the estimated phi and PCA3 thresholds were 22.4 and 7.6, respectively. Their corresponding empirical specificities were $24.6 \%$ and $17.4 \%$, which became $24.1 \%$ and $16.1 \%$ upon oscillating bias correction. For each biomarker, various $95 \%$ confidence intervals for the specificity were constructed. The original Zhou-Qin interval, via resampling bootstrap, exhibited considerable variability under both resampling sizes of 200 and 500. Other intervals also had appreciable differences from each other. Paired comparison was made between phi and PCA3, and 95\% confidence intervals were constructed for their difference in specificity at controlled 95\% sensitivity. The score intervals were tighter than the Wald and percentile ones, which is consistent with the simulation results.

To contrast, areas under the ROC curves were also estimated. They were 0.792 (95\% confidence interval: $0.750-0.835)$ and 0.696 (95\% confidence interval: $0.647-0.744)$ for phi and PCA3, respectively. The difference was statistically significant with a p-value of 0.003 . While area under the 
ROC curve is a commonly used accuracy metric, its interpretation is different. To echo Section 1, specificity at controlled $95 \%$ sensitivity is clinically more sensible in this application.

\section{Discussion}

In this article, we have investigated interval estimation for specificity at a controlled sensitivity level. Exact bootstrap is advocated over kernel smoothing and resampling bootstrap for the inference. Furthermore, we have proposed novel score confidence intervals, which showed competitive or superior performance in comparison with existing ones in single-biomarker evaluation and two-biomarker comparison.

We have limited our scope to confidence intervals on the basis of the empirical specificity at controlled sensitivity or its variants, for their robustness. However, there is an extensive literature on kernel-based estimators of the ROC curve, including Zou et al. (1997) and Lloyd (1998). Lloyd and Yong (1999) showed that such estimators have smaller mean squared error asymptotically than the empirical estimator, provided proper choice of the smoothing bandwidth. Hall et al. (2004) investigated the confidence intervals based on the kernel-smoothed ROC curve as well as kernel-based variance estimation. Theoretically this approach could lead to accuracy 
gain. Nevertheless, appropriate selection of a multitude of smoothing parameters is required and further development would be needed for wide practical adoption.

\section{Supplementary Materials}

Additional simulation results, with unequal case and controls sizes, are available in the supplementary material. They are for both single-biomarker evaluation and two-biomarker comparison.

\section{Acknowledgment}

The authors thank the reviewers for helpful comments and suggestions that have led to an improvement in the presentation. Support by grants CA230268, CA113913, and AI050409 from the US National Institutes of Health is gratefully acknowledged. 


\section{Appendix: Proofs and other technical details}

\section{Proof of Lemma 1}

Since $n F^{*}(x) \sim \operatorname{Binomial}\{n, \widehat{F}(x)\}$ given the data,

$$
\begin{aligned}
\operatorname{Pr}^{*}\left\{F^{*}(x) \geq p\right\} & =\operatorname{Pr}^{*}\left\{n F^{*}(x) \geq\lceil n p\rceil\right\} \\
& =\sum_{k=\lceil n p\rceil}^{n}\left(\begin{array}{l}
n \\
k
\end{array}\right) \widehat{F}(x)^{k}\{1-\widehat{F}(x)\}^{n-k} \\
& =\lceil n p\rceil\left(\begin{array}{c}
n \\
\lceil n p\rceil
\end{array}\right) \int_{0}^{\widehat{F}(x)} y^{\lceil n p\rceil-1}(1-y)^{n-\lceil n p\rceil} d y \\
& =\operatorname{Pr}^{*}\{B \leq \widehat{F}(x)\},
\end{aligned}
$$

where the third equality follows by induction from $n$ downward as the value of $\lceil n p\rceil$. By a basic result of quantile function (e.g., Serfling 1980, lemma 1.1.4), $F^{*}(x) \geq p$ if and only if $F^{*-1}(p) \leq x$, and $B \leq \widehat{F}(x)$ if and only if $\widehat{F}^{-1}(B) \leq x$. The assertion then follows.

\section{Proof of Theorem 1}

Write $D[a, b]$ as the space of cadlag functions in $[a, b]$ with some $a$ and $b$ such that $a<\tau_{0}<b$. Endow such a space with the supremum norm and their product with the max supremum norm. In light of $\widehat{\phi}=\widehat{F}_{\circ}\left[\widehat{F}_{\bullet}^{-1}\{(1-\right.$ $\left.\left.\left.\rho_{0}\right)+\right\}-\right], \widehat{\phi}$ is the plug-in estimator in the map $\left\{F_{\bullet}, F_{\circ}\right\} \mapsto \phi_{0}$ decomposed 
as

$$
\left.\begin{array}{l}
F_{\bullet} \in D[a, b] \mapsto \tau_{0} \in R \\
F_{\circ} \in D[a, b]
\end{array}\right\} \mapsto \phi_{0} \in R
$$

With $F_{\bullet}$ being differentiable with positive derivative at $\tau_{0}, F_{\bullet} \mapsto \tau_{0}$ is

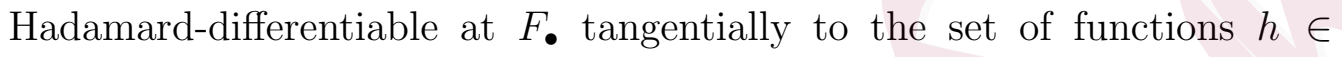
$D[a, b]$ that are continuous at $\tau_{0}$, with derivative $-h\left(\tau_{0}\right) / F_{\bullet}^{\prime}\left(\tau_{0}\right)($ e.g., van der Vaart, 1998, lemma 21.3). Meanwhile, given $F_{\circ}$ being differentiable at $\tau_{0}$, it can be shown that $\left(\tau_{0}, F_{\circ}\right) \mapsto \phi_{0}$ is Hadamard-differentiable at $\left(\tau_{0}, F_{\circ}\right)$ tangentially to the set $\{k: \in R\} \times\left\{l: \in D[a, b]\right.$, continuous at $\left.\tau_{0}\right\}$, with derivative $F_{\circ}^{\prime}\left(\tau_{0}\right) k+l\left(\tau_{0}\right)$. By the chain rule, $\left\{F_{\bullet}, F_{\circ}\right\} \mapsto \phi_{0}$ is then Hadamarddifferentiable at $\left\{F_{\bullet}, F_{\circ}\right\}$ tangentially to the set $\left\{h: \in D[a, b]\right.$, continuous at $\left.\tau_{0}\right\} \times$ $\left\{l: \in D[a, b]\right.$, continuous at $\left.\tau_{0}\right\}$, with derivative $-h\left(\tau_{0}\right) F_{\circ}^{\prime}\left(\tau_{0}\right) / F_{\bullet}^{\prime}\left(\tau_{0}\right)+$ $l\left(\tau_{0}\right)$. As the Hadamard-differentiability implies continuity, $\widehat{\phi}$ is strongly consistent for $\phi_{0}$ following the strong consistency of $\left\{\widehat{F}_{\bullet}, \widehat{F}_{\circ}\right\}$ for $\left\{F_{\bullet}, F_{\circ}\right\}$ by the Glivenko-Cantelli theorem. With the weak convergence of $n_{\bullet}^{1 / 2}\left(\widehat{F}_{\bullet}-F_{\bullet}\right)$ and $n_{\circ}^{1 / 2}\left(\widehat{F}_{\circ}-F_{\circ}\right), \widehat{\phi}$ is $A N\left(\phi_{0}, \sigma^{2}\right)$ following the functional delta method and the asymptotic normality of $\left\{\widehat{F}_{\bullet}\left(\tau_{0}\right), \widehat{F}_{\circ}\left(\tau_{0}\right)\right\}$ by the central limit theorem. Furthermore, by the results on empirical bootstrap and delta method for bootstrap (van der Vaart, 1998, theorems 23.7 and 23.9), the conditional distribution of $n_{\circ}^{1 / 2}\left(\phi^{*}-\widehat{\phi}\right)$ given the observed data converges in distribution 
to the same limit as $n_{\circ}^{1 / 2}\left(\widehat{\phi}-\phi_{0}\right)$.

To investigate the bootstrap variance estimator, $\widehat{F}_{\circ}\left(\tau^{*}-\right)$ is an important building block as shown in (2.4). Write $\phi(\rho)=F_{\circ}\left[F_{\bullet}^{-1}\{(1-\rho)+\}-\right]$ and $\widehat{\phi}(\rho)=\widehat{F}_{\circ}\left[\widehat{F}_{\bullet}^{-1}\{(1-\rho)+\}-\right]$; of course, $\phi_{0} \equiv \phi\left(\rho_{0}\right)$ and $\widehat{\phi} \equiv \widehat{\phi}\left(\rho_{0}\right)$. By Lemma $1, \widehat{F}_{\circ}\left(\tau^{*}-\right)$ is equivalent to $\widehat{\phi}\left(B_{\bullet}\right)$ in conditional distribution. As a fact, $B_{\bullet}$ has the same distribution as $C_{1} /\left(C_{1}+C_{2}\right)$ with $C_{1} \sim \chi^{2}\left(2 n_{\bullet}-2 r+\right.$ 2), $C_{2} \sim \chi^{2}(2 r)$, and $C_{1} \Perp C_{2}$. Therefore, $B$ • converges almost surely to $\rho_{0}$ by strong law of large numbers and continuous mapping theorem, and $n_{\bullet}^{1 / 2}\left(B_{\bullet}-\rho_{0}\right)$ converges in distribution to $N\left\{0, \rho_{0}\left(1-\rho_{0}\right)\right\}$ by the central limit theorem and delta method. Furthermore, we give a bound on the tail probability of $B$. on the basis of the sub-Gaussianity of the Beta distribution (Marchal and Arbel, 2017). Since both $B \bullet$ and $1-B_{\bullet}$ are $\left\{4\left(n_{\bullet}+2\right)\right\}^{-1}$ sub-Gaussian,

$$
\operatorname{Pr}\left\{\left|B_{\bullet}-E\left(B_{\bullet}\right)\right|>b\right\} \leq 2 \exp \left\{-2\left(n_{\bullet}+2\right) b^{2}\right\}
$$

for any constant $b$.

The asymptotic normality result on $\widehat{\phi}$ can be extended to the weak convergence of $\widehat{\phi}(\rho)$ in a neighborhood of $\rho$ around $\rho_{0}$ under the additional condition (iv). The arguments are essentially the same, upon appropriate modifications of domain and range spaces of the functions involved and with extended result on Hadamard differentiability of the quantile function 
(van der Vaart, 1998, lemma 21.4). Given that $n_{\circ}^{1 / 2}\{\widehat{\phi}(\rho)-\phi(\rho)\}$ converges weakly to a Gaussian process in a neighborhood of $\rho$ around $\rho_{0}$, for any $d_{n \circ} \downarrow 0$, one can show

$$
\sup _{|d| \leq d_{n_{\circ}}}\left|\widehat{\phi}\left(\rho_{0}+d\right)-\widehat{\phi}\left(\rho_{0}\right)-\phi\left(\rho_{0}+d\right)+\phi\left(\rho_{0}\right)\right|=o_{p}\left(n_{\circ}^{-1 / 2}\right)
$$

see, for example, Huang (2017, appendix). Meanwhile, the differentiability of $\phi(\rho)$ at $\rho_{0}$ implies

$$
\sup _{|d| \leq d_{n_{\circ}}} d^{-1}\left|\phi\left(\rho_{0}+d\right)-\phi\left(\rho_{0}\right)-\phi^{\prime}\left(\rho_{0}\right) d\right|=o(1)
$$

where $\phi^{\prime}\left(\rho_{0}\right)=-F_{\circ}^{\prime}\left(\tau_{0}\right) / F_{\bullet}^{\prime}\left(\tau_{0}\right)$.

Let $c_{n_{\bullet}}=\left\{\log n_{\bullet} /\left(n_{\bullet}+2\right)\right\}^{1 / 2}$. Following $(\mathrm{A} .12), \operatorname{Pr}\left\{\left|B_{\bullet}-E\left(B_{\bullet}\right)\right|>\right.$ $\left.c_{n_{\bullet}}\right\} \leq 2 n_{\bullet}^{-2}$. Given $E\left(B_{\bullet}\right)-\rho_{0}=O\left(n_{\bullet}^{-1}\right),\left|B_{\bullet}-\rho_{0}\right|=\left|B_{\bullet}-E\left(B_{\bullet}\right)\right|+O\left(n_{\bullet}^{-1}\right)$. Note that $E_{*}$ takes expectation over $B_{\bullet}$. Then,

$$
\begin{aligned}
E_{*}\left\{\widehat{\phi}\left(B_{\bullet}\right)\right\}= & E_{*}\left[\widehat{\phi}\left(B_{\bullet}\right) I\left\{\left|B_{\bullet}-E\left(B_{\bullet}\right)\right| \leq c_{n_{\bullet}}\right\}\right]+O\left(n_{\bullet}^{-2}\right) \\
= & \widehat{\phi}\left(\rho_{0}\right)+\phi^{\prime}\left(\rho_{0}\right) E\left[\left(B_{\bullet}-\rho_{0}\right) I\left\{\left|B_{\bullet}-E\left(B_{\bullet}\right)\right| \leq c_{n_{\bullet}}\right\}\right] \\
& +o_{p}\left\{n_{\bullet}^{-1 / 2}+E\left|B_{\bullet}-\rho_{0}\right|\right\} \\
= & \widehat{\phi}+o_{p}\left(n_{\bullet}^{-1 / 2}\right),
\end{aligned}
$$

since $E\left|B_{\bullet}-E\left(B_{\bullet}\right)\right|=O\left(n_{\bullet}^{-1 / 2}\right)$ following $\operatorname{Var}\left(B_{\bullet}\right)=O\left(n_{\bullet}^{-1}\right)$ by Jensen's inequality. Similarly, $E_{*}\left\{\widehat{\phi}\left(B_{\bullet}\right)^{2}\right\}=\widehat{\phi}^{2}+o_{p}\left(n_{\bullet}^{-1 / 2}\right)$. Therefore, $n_{\circ} \widehat{\sigma}_{2}^{2}=$ 
$E_{*}\left[\widehat{\phi}\left(B_{\bullet}\right)\left\{1-\widehat{\phi}\left(B_{\bullet}\right)\right\}\right]$ converges to $n_{\circ} \sigma_{2}^{2}$ in probability. By similar arguments,

$$
\begin{aligned}
\widehat{\sigma}_{1}^{2} & =E_{*}\left[\left\{\widehat{\phi}\left(B_{\bullet}\right)-\widehat{\phi}+o_{p}\left(n_{\bullet}^{-1 / 2}\right)\right\}^{2}\right] \\
& =E\left[\left\{\phi^{\prime}\left(\rho_{0}\right)\left(B_{\bullet}-\rho_{0}\right)\right\}^{2} I\left\{\left|B_{\bullet}-E\left(B_{\bullet}\right)\right| \leq c_{n_{\bullet}}\right\}\right]+o\left(n_{\bullet}^{-1}\right) \\
& =\sigma_{1}^{2}+o\left(n_{\bullet}^{-1}\right) .
\end{aligned}
$$

Then, $n_{\bullet} \widehat{\sigma}_{1}^{2}$ converges to $n_{\bullet} \sigma_{1}^{2}$ in probability. Thus, $n_{\circ} \widehat{\sigma}^{2}$ converges to $n_{\circ} \sigma^{2}$ in probability as well.

\section{Proof of Theorem 2}

The arguments for the proof of Theorem 1 with a single biomarker extend in a straightforward fashion to the two-biomarker problem, for the estimation with correlated specificities at a common controlled sensitivity level. Subsequently, the claims on the difference in specificity follow.

\section{Bias analysis of $\widehat{\phi}$ in Section 4}

Following Remark 1, $\widehat{\tau}$ has the same distribution as $F_{\bullet}^{-1}\left(1-B_{\bullet}\right)$. Thus, $E(\widehat{\phi})=E\{E(\widehat{\phi} \mid \widehat{\tau})\}=E\left\{F_{\circ}(\widehat{\tau})\right\}=E\left\{\phi\left(B_{\bullet}\right)\right\}$. In light of (A.12) and with 


$$
\begin{aligned}
& c_{n_{\bullet}}=\left\{\log n_{\bullet} /\left(n_{\bullet}+2\right)\right\}^{1 / 2}, \\
& E\left\{\phi\left(B_{\bullet}\right)\right\}=E\left\{\phi\left(B_{\bullet}\right) I\left(\left|B_{\bullet}-E\left(B_{\bullet}\right)\right| \leq c_{n_{\bullet}}\right)\right\}+O\left(n_{\bullet}^{-2}\right) \\
& =\phi\left\{E\left(B_{\bullet}\right)\right\}+\phi^{\prime}\left\{E\left(B_{\bullet}\right)\right\} E\left[\left\{B_{\bullet}-E\left(B_{\bullet}\right)\right\} I\left(\left|B_{\bullet}-E\left(B_{\bullet}\right)\right| \leq c_{n_{\bullet}}\right)\right] \\
& +E\left\{\left[\phi^{\prime \prime}\left\{E\left(B_{\bullet}\right)\right\} / 2+o(1)\right]\left\{B_{\bullet}-E\left(B_{\bullet}\right)\right\}^{2} I\left(\left|B_{\bullet}-E\left(B_{\bullet}\right)\right| \leq c_{n_{\bullet}}\right)\right\} \\
& +O\left(n_{\bullet}^{-2}\right) \\
& =\phi\left\{E\left(B_{\bullet}\right)\right\}+\left\{\phi^{\prime \prime}\left(\rho_{0}\right) / 2+o(1)\right\} E\left[\left\{B_{\bullet}-E\left(B_{\bullet}\right)\right\}^{2}\right]+O\left(n_{\bullet}^{-2}\right) \\
& =\phi_{0}+\phi^{\prime}\left(\rho_{0}\right)\left(1-\frac{r}{n_{\bullet}+1}-\rho_{0}\right)+\phi^{\prime \prime}\left(\rho_{0}\right) \frac{\rho_{0}\left(1-\rho_{0}\right)}{2 n_{\bullet}}+o\left(n_{\bullet}^{-1}\right) \text {, }
\end{aligned}
$$

which gives equation (4.10). The second equation above is an application of Taylor expansion, with the existence and continuity of $\phi^{\prime \prime}(\cdot)$ in a neighborhood of $\rho_{0}$ under the assumptions given.

\section{References}

Agresti, A. and Coull, B. A. (1998). Approximate is better than "exact" for interval estimation of binomial proportions. The American Statistician 52, 119-126.

Brown, L. D., Cai, T. T., and DasGupta, A. (2001). Interval estimation of a binomial proportion. Statistical Science 16, 101-133.

Brown, L. D., Cai, T. T., and DasGupta, A. (2002). Confidence intervals for a binomial proportion and asymptotic expansions. Annals of 
Statistics 30, 160-201.

Catalona, W. J., Partin, A. W., Slawin, K. M., et al. (1998). Use of the percentage of free prostate-specific antigen to enhance differentiation of prostate cancer from benign prostatic disease: A prospective multicenter clinical trial. JAMA 279, 1542-1547.

Clopper, C. J. and Pearson, E. S. (1934). The use of confidence or fiducial limits illustrated in the case of the binomial. Biometrika 26, 404-413.

Efron, B. (1979). Bootstrap methods: Another look at the Jackknife. Annals of Statistics 7, 1-26.

Efron B. and Tibshirani, R. J. (1994). An Introduction to the Bootstrap. New York: Chapman \& Hall/CRC.

Ghosh, M., Parr, W. C., Singh, K., and Babu, G. J. (1984). A note on bootstrapping the sample median. Annals of Statistics 12, 1130-1135.

Greenhouse, S. W. and Mantel, N. (1950). The evaluation of diagnostic tests. Biometrics 6, 399-412.

Hall, P., Hyndman, R. J., and Fan, Y. (2004). Nonparametric confidence intervals for receiver operating characteristic curves. Biometrika 91, $743-750$.

Hall, P. and Martin, M. A. (1991). On the error incurred using the bootstrap variance estimate when constructing confidence intervals for 
quantiles. Journal of Multivariate Analysis 38, 70-81.

Hsieh, F. and Turnbull, B. W. (1996). Nonparametric and semiparametric estimation of the receiver operating characteristic curve. Annals of Statistics 24, 25-40.

Huang, Y. (2017). Restoration of monotonicity respecting in dynamic regression. Journal of the American Statistical Association 112, 613622.

Ioannidis, J. P. and Panagiotou, O. A. (2011). Comparison of effect sizes associated with biomarkers reported in highly cited individual articles and in subsequent meta-analyses. JAMA 305, 2200-2210.

Linnet, K. (1987). Comparison of quantitative diagnostic tests: type I error, power, and sample size. Statistics in Medicine 6, 147-158.

Lloyd, C. J. (1998). The use of smoothed ROC curves to summarise and compare diagnostic systems. Journal of the American Statistical Association 93, 1356-1364.

Lloyd, C. J. and Yong, Z. (1999). Kernel estimators of the ROC curve are better than empirical. Statistics \& Probability Letters 44, 221-228.

Marchal, O. and Arbel, J. (2017). On the sub-Gaussianity of the Beta and Dirichlet distributions. Electronic Communications in Probability 54, $1-14$. 
Maritz, J. S. and Jarrett, R. G. (1978). A note on estimating the variance of the sample median. Journal of the American Statistical Association 73, 194-196.

Pepe, M. S. (2003). The Statistical Evaluation of Medical Tests for Classification and Prediction. Oxford: Oxford University Press.

Platt, R. W., Hanley, J. A., and Yang, H. (2000). Bootstrap confidence intervals for the sensitivity of a quantitative diagnostic test. Statistics in Medicine 19, 313-322.

Sanda, M. G., Feng, Z., Howard, D. H., et al. (2017). Association between combined TMPRSS2:ERG and PCA3 RNA urinary testing and detection of aggressive prostate cancer. JAMA Oncology 3, 1085-1093.

Serfling, R. J. (1980). Approximation Theorems of Mathematical Statistics. New York: John Wiley \& Sons.

Shao, J. (1990). Bootstrap estimation of the asymptotic variances of statistical functionals. Annals of the Institute of Statistical Mathematics 42, 737-752.

Silverman, B. (1986). Density Estimation for Statistics and Data Analysis. London: Chapman and Hall.

Tzoulaki, I., Siontis, K. C., and Ioannidis, J. P. (2011). Prognostic effect size of cardiovascular biomarkers in datasets from observational stud- 
ies versus randomised trials: meta-epidemiology study. BMJ $\mathbf{3 4 3}$, d6829.

van der Vaart, A. W. (1998). Asymptotic Statistics. New York: Cambridge University Press.

Wilson, E. B. (1927). Probable inference, the law of succession, and statistical inference. Journal of the American Statistical Association 22, 209-212.

Zhou, X.-H. and Qin, G. (2005). Improved confidence intervals for the sensitivity at a fixed level of specificity of a continuous-scale diagnostic test. Statistics in Medicine 24, 465-477.

Zou, K. H., Hall, W. J., and Shapiro, D. E. (1997). Smooth non-parametric receiver operating characteristic (ROC) curves for continuous diagnostic tests. Statistics in Medicine 16, 2143-2156.

Department of Biostatistics and Bioinformatics, Emory University

E-mail: yhuang5@emory.edu

Ann \& Robert H. Lurie Children's Hospital of Chicago

E-mail: iparakati@luriechildrens.org

Department of Urology, Emory University

E-mail: dattatraya.patil@emory.edu, martinsanda@emory.edu 
Table 1: Simulation summary statistics of exact bootstrap-based $95 \%$ confidence intervals for difference in specificity at controlled $95 \%$ sensitivity in two-biomarker comparison.

\begin{tabular}{|c|c|c|c|c|c|c|c|c|c|c|c|c|c|}
\hline \multirow[b]{3}{*}{$\phi_{0 X}$} & \multirow[b]{3}{*}{$\delta_{0}$} & \multirow[b]{3}{*}{ size } & & \multicolumn{5}{|c|}{ unpaired biomarkers } & \multicolumn{5}{|c|}{ paired biomarkers } \\
\hline & & & & \multicolumn{2}{|c|}{ Wald } & \multirow[t]{2}{*}{ Pct } & \multicolumn{2}{|c|}{ Score } & \multicolumn{2}{|c|}{ Wald } & \multirow[t]{2}{*}{ Pct } & \multicolumn{2}{|c|}{ Score } \\
\hline & & & & $\widehat{\phi}$ & $\widetilde{\phi}$ & & $\widehat{\phi}$ & $\widetilde{\phi}$ & $\widehat{\phi}$ & $\widetilde{\phi}$ & & $\widehat{\phi}$ & $\widetilde{\phi}$ \\
\hline \multirow[t]{4}{*}{0.2} & 0.0 & 50 & $\mathrm{C}$ & 966 & 968 & 987 & 976 & 979 & 979 & 978 & 991 & 982 & 986 \\
\hline & & & $\mathrm{L}$ & 579 & 579 & 576 & 467 & 445 & 516 & 516 & 515 & 430 & 409 \\
\hline & & 200 & $\mathrm{C}$ & 950 & 951 & 975 & 956 & 955 & 959 & 956 & 979 & 963 & 958 \\
\hline & & & $\mathrm{L}$ & 293 & 293 & 292 & 275 & 268 & 261 & 261 & 261 & 248 & 242 \\
\hline \multirow[t]{8}{*}{0.4} & 0.2 & 50 & $\mathrm{C}$ & 956 & 963 & 985 & 970 & 970 & 964 & 974 & 986 & 970 & 965 \\
\hline & & & $\mathrm{L}$ & 671 & 671 & 663 & 540 & 525 & 600 & 600 & 595 & 500 & 486 \\
\hline & & 200 & $\mathrm{C}$ & 939 & 942 & 966 & 942 & 944 & 948 & 949 & 972 & 955 & 953 \\
\hline & & & $\mathrm{L}$ & 339 & 339 & 337 & 318 & 313 & 303 & 303 & 302 & 288 & 284 \\
\hline & 0.0 & 50 & $\mathrm{C}$ & 964 & 958 & 981 & 968 & 962 & 968 & 974 & 993 & 971 & 978 \\
\hline & & & $\mathrm{L}$ & 749 & 749 & 744 & 594 & 584 & 658 & 658 & 657 & 545 & 535 \\
\hline & & 200 & $\mathrm{C}$ & 938 & 933 & 963 & 941 & 935 & 946 & 940 & 972 & 951 & 945 \\
\hline & & & $\mathrm{L}$ & 380 & 380 & 378 & 355 & 352 & 337 & 337 & 336 & 319 & 317 \\
\hline \multirow[t]{12}{*}{0.6} & 0.4 & 50 & $\mathrm{C}$ & 955 & 956 & 984 & 946 & 947 & 962 & 968 & 989 & 955 & 958 \\
\hline & & & $\mathrm{L}$ & 671 & 674 & 668 & 556 & 551 & 607 & 608 & 605 & 517 & 512 \\
\hline & & 200 & $\mathrm{C}$ & 955 & 953 & 971 & 959 & 942 & 964 & 955 & 980 & 961 & 957 \\
\hline & & & $\mathrm{L}$ & 339 & 339 & 337 & 321 & 319 & 306 & 306 & 304 & 293 & 291 \\
\hline & 0.2 & 50 & $\mathrm{C}$ & 957 & 959 & 981 & 957 & 955 & 964 & 970 & 991 & 963 & 969 \\
\hline & & & $\mathrm{L}$ & 752 & 753 & 751 & 601 & 600 & 663 & 663 & 664 & 552 & 552 \\
\hline & & 200 & $\mathrm{C}$ & 944 & 941 & 968 & 949 & 943 & 961 & 959 & 979 & 966 & 957 \\
\hline & & & $\mathrm{L}$ & 381 & 381 & 379 & 356 & 356 & 339 & 339 & 337 & 321 & 321 \\
\hline & 0.0 & 50 & $\mathrm{C}$ & 962 & 960 & 980 & 959 & 956 & 970 & 964 & 993 & 966 & 962 \\
\hline & & & $\mathrm{L}$ & 760 & 760 & 765 & 599 & 609 & 669 & 669 & 675 & 551 & 560 \\
\hline & & 200 & $\mathrm{C}$ & 956 & 949 & 970 & 954 & 949 & 964 & 959 & 983 & 966 & 959 \\
\hline & & & $\mathrm{L}$ & 380 & 380 & 379 & 355 & 358 & 339 & 339 & 338 & 321 & 324 \\
\hline \multirow[t]{16}{*}{0.8} & 0.6 & 50 & $\mathrm{C}$ & 960 & 959 & 980 & 927 & 928 & 967 & 969 & 979 & 927 & 932 \\
\hline & & & $\mathrm{L}$ & 582 & 586 & 592 & 519 & 522 & 537 & 539 & 544 & 486 & 488 \\
\hline & & 200 & $\mathrm{C}$ & 953 & 950 & 969 & 946 & 944 & 960 & 956 & 971 & 949 & 944 \\
\hline & & & $\mathrm{L}$ & 290 & 290 & 288 & 281 & 281 & 264 & 264 & 263 & 257 & 258 \\
\hline & 0.4 & 50 & $\mathrm{C}$ & 957 & 962 & 973 & 936 & 939 & 968 & 969 & 978 & 936 & 942 \\
\hline & & & $\mathrm{L}$ & 679 & 679 & 685 & 562 & 569 & 608 & 608 & 612 & 519 & 526 \\
\hline & & 200 & $\mathrm{C}$ & 956 & 949 & 966 & 947 & 948 & 961 & 957 & 979 & 960 & 954 \\
\hline & & & $\mathrm{L}$ & 337 & 337 & 335 & 319 & 321 & 305 & 305 & 304 & 291 & 293 \\
\hline & 0.2 & 50 & $\mathrm{C}$ & 957 & 963 & 979 & 944 & 958 & 965 & 966 & 985 & 953 & 951 \\
\hline & & & $\mathrm{L}$ & 690 & 690 & 701 & 552 & 569 & 614 & 614 & 622 & 510 & 526 \\
\hline & & 200 & $\mathrm{C}$ & 961 & 960 & 967 & 956 & 947 & 961 & 962 & 979 & 963 & 967 \\
\hline & & & $\mathrm{L}$ & 337 & 337 & 336 & 316 & 321 & 304 & 304 & 303 & 288 & 293 \\
\hline & 0.0 & 50 & $\mathrm{C}$ & 970 & 961 & 989 & 957 & 946 & 971 & 971 & 990 & 970 & 969 \\
\hline & & & $\mathrm{L}$ & 619 & 619 & 639 & 492 & 519 & 551 & 551 & 569 & 454 & 480 \\
\hline & & 200 & $\mathrm{C}$ & 965 & 956 & 975 & 962 & 952 & 974 & 967 & 983 & 967 & 963 \\
\hline & & & $\mathrm{L}$ & 289 & 289 & 289 & 272 & 279 & 258 & 258 & 259 & 245 & 252 \\
\hline
\end{tabular}




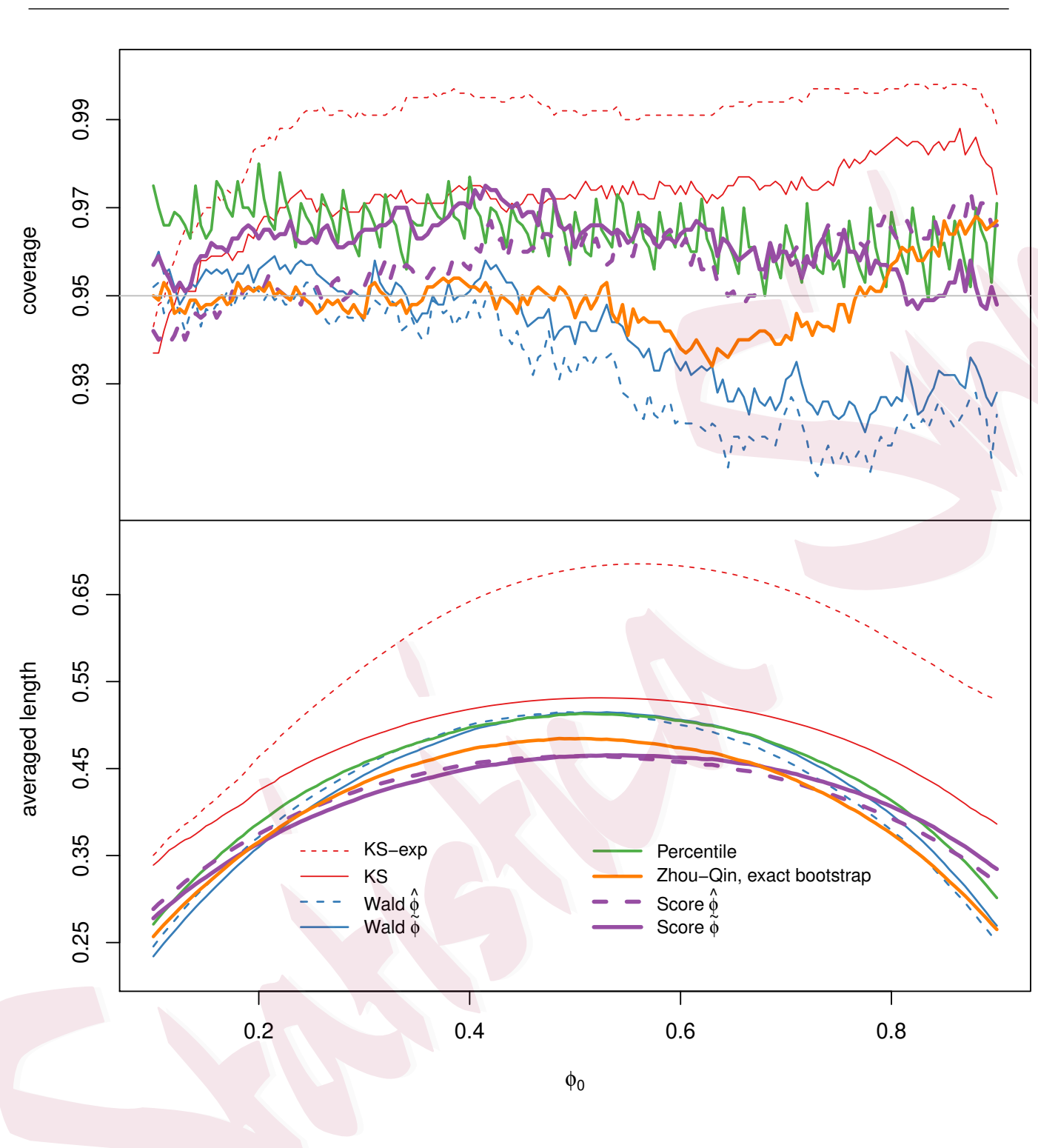

Figure 1: Simulation summaries of $95 \%$ confidence intervals for specificity at controlled $95 \%$ sensitivity in single-biomarker evaluation, under fixed sizes $n_{\bullet}=n_{\circ}=50$ and variable $\phi_{0}$. KS is the kernel smoothing-based Wald confidence intervals as in Pepe (2003), whereas KS-exp corresponds to that applied to exponentially transformed biomarker data. 

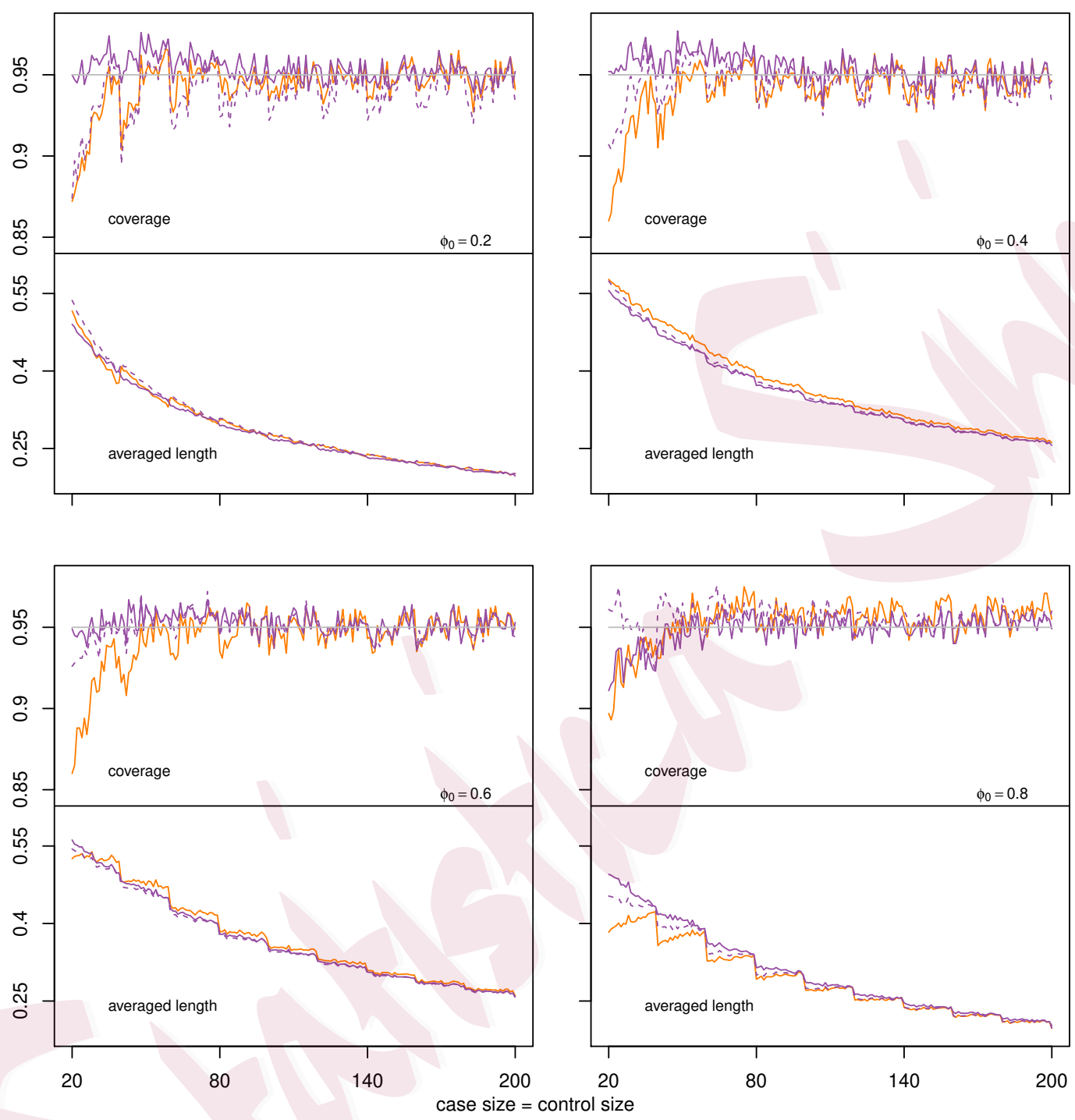

Figure 2: Simulation summaries of $95 \%$ confidence intervals for specificity at controlled $95 \%$ sensitivity in single-biomarker evaluation, under fixed $\phi_{0}$ and variable sizes $n_{\bullet}=n_{\circ}$. Score intervals, based on $\widehat{\phi}$ and $\widetilde{\phi}$, and the exact bootstrap-based Zhou-Qin are included, with the same labeling as in Figure 1. 

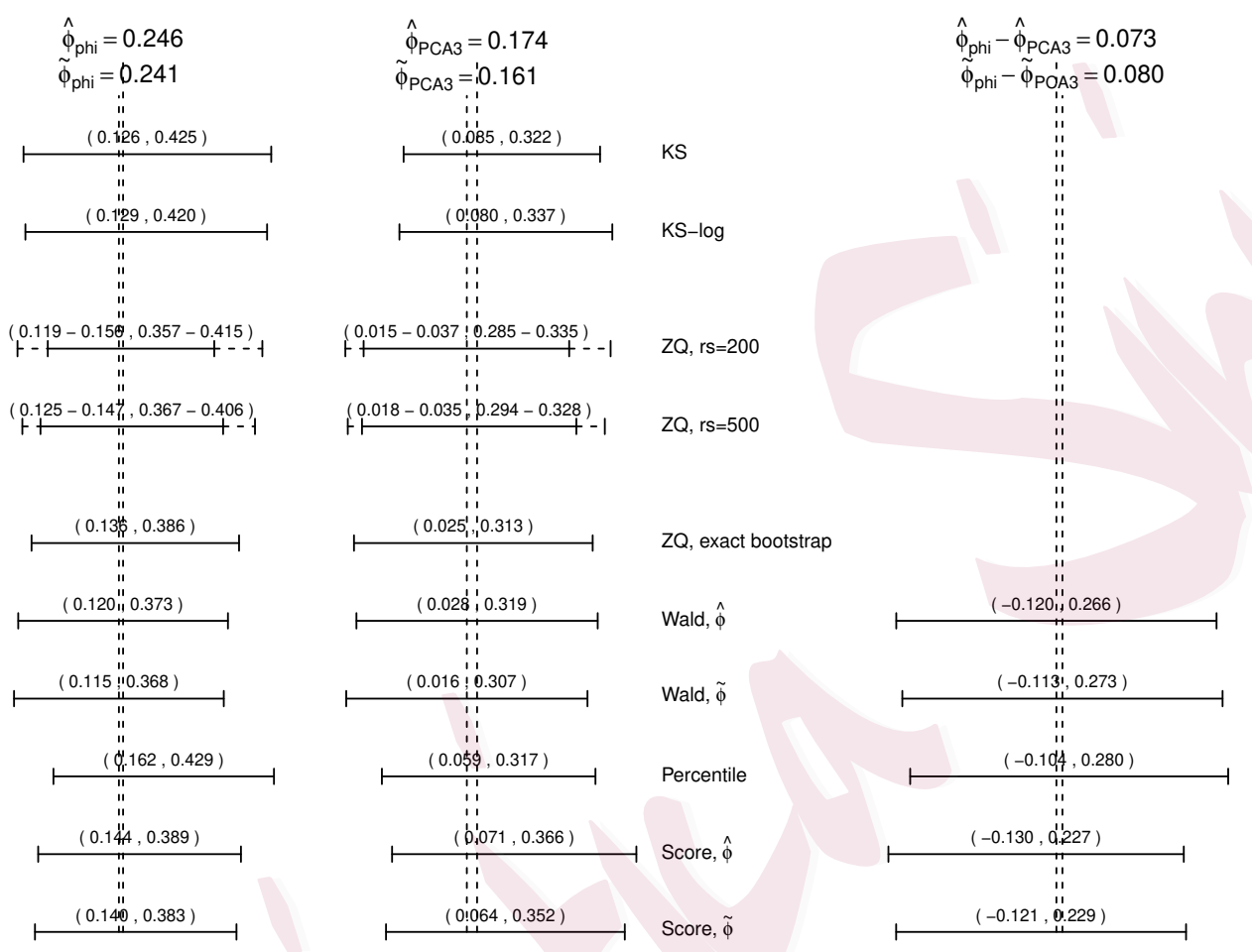

Figure 3: Analysis results of the prostate cancer study: point estimates and 95\% confidence intervals of specificity at 95\% sensitivity for phi and PCA3 as diagnostic biomarkers for aggressive prostate cancer. KS and KS-log are the kernel smoothing-based Wald confidence intervals, as applied to untransformed and logarithm-transformed biomarker data, respectively. For the resampling Zhou-Qin (ZQ) confidence interval with a given resampling size (rs), ranges of the left and right bounds over 100 runs are provided. 